\title{
The upgrade of ID29: a novel instrument for Time resolved serial crystallography experiment at the ESRF Extremely Brilliant Source
}

\author{
Daniele de Sanctis \\ ESRF - The European Synchrotron, 71 av des Martyrs, 38000 Grenoble, France \\ daniele.de_sanctis@esrf.fr
}

During the last decades, structural biology had a major impact in understanding the structure-functional aspects of some of the most important biological machineries. The new ESRF Extremely Brilliant Source opened a new age in microcrystallography and permitted to extend further the capabilities of the macromolecular crystallography beamlines and will open new pathways in the study of timedependent structural changes. This is the scope of the upgrade of the ID29 beamline.

The new beamline combined cutting edge instrumentations to fully exploit serial crystallography experiments at room temperature. This presentaition will present the the beamline design with particular relevance to the new instrumentations and present the new scientifc opportunities that it will offer to the structural biology user community. 